\title{
Safety and reliability of electrical insulation
}

\author{
Miguel Castro Fernández ${ }^{1}$
}

${ }^{1}$ Ciudad Universitaria José Antonio Echeverría (CUJAE), Havana, Cuba.

Email: mcastro@electrica.cujae.edu.cu

Received: December 30 ${ }^{\text {th }}, 2017$.

\begin{abstract}
The insulators, in their daily operation, are subject to the action of the environment and their own exploitation, being understood between the latter the tensions at the frequency of the system and the surges that originate from different causes. In the case of the environment are two fundamental factors that cause the insulator to lose its insulating characteristics: the accumulation of polluting particles on the surface of the same that when it is wetted by light rain or fog becomes conductive, to such an extent that an arc of current, or discharge, is established between the structure that supports the insulators and the conductors, damaging the whole assembly and causing cuts or interruptions in the transmission or distribution of energy, causing reliability problems of the service, on the other hand, The metal parts of the insulators are affected by corrosion problems affecting their mechanical characteristics. Industrial users demand high reliability services, which not only comply with certain voltage and frequency ranges but also offer continuity in service; Among the processes that are most affected by the cuts in the service are those of automatic industrialization, because they are increasingly sensitive and can alter a process with a minimum interruption in service and causing millions in losses. Determine when to take corrective actions to anticipate transient or permanent failures in high voltage insulators has been a problem for companies in the electricity sector for many years; In the case of pollution, the solution has been towards the replacement of the insulation or the execution of periodic washings of the same, sometimes without any technical criteria, which demands a considerable workforce. In the case of corrosion or the partial or total destruction of insulation by surges, companies have no choice but to replace the insulation when the failure occurs, in general, or what is known as the application of corrective maintenance. In recent years, this problem has become more important as a result of the free competition of companies in the electricity sector, since it requires an optimization of them and therefore a reduction in maintenance and labor costs. The present work tries to expose the close relationship that exists between isolation and reliability from the new paradigms that appear in the maintenance activity, which offer some interesting changes in the environment until now seen in this direction.
\end{abstract}

Accepted: January $18^{\text {th }}, 2018$.

Keywords: Insulators, maintenance, reliability.

\section{RESUMEN}

\section{Seguridad y confiabilidad del aislamiento eléctrico}

Los aisladores, en su operación diaria, están sometidos a la acción del medio ambiente y de su propia explotación entendiéndose entre estas últimas las tensiones a la frecuencia del sistema y las sobretensiones que se originan por diferentes causas. En el caso del medio ambiente son dos factores fundamentales los que provocan que el aislador vaya perdiendo sus características aislantes: la acumulación de partículas contaminantes sobre la superficie de los mismos que cuando es humedecida por lluvias ligeras o niebla llega a ser conductora, a tal grado que se establece un arco de corriente, o descarga, entre la estructura que soporta los aisladores y los conductores, dañando a todo el conjunto y ocasionando cortes o interrupciones de la transmisión o distribución de energía ocasionando problemas de confiabilidad del servicio , por otro lado, las partes metálicas de los aisladores son afectadas por problemas de corrosión afectando sus características mecánicas. Los usuarios industriales demandan servicios de alta confiabilidad, que no sólo cumplan con ciertos rangos de tensión y frecuencia sino que también ofrezcan una continuidad en el servicio; entre los procesos que más son afectados por los cortes en el servicio están los de industrialización automática, debido a que son cada día más sensibles pudiendo alterar un proceso con una mínima interrupción en el servicio y ocasionando pérdidas millonarias. Determinar cuando tomar acciones correctivas para prever los fallos transitorios o permanentes en los aisladores de alta tensión ha sido un problema de las empresas del sector eléctrico por muchos años; en el caso de la contaminación, la solución ha estado hacia la sustitución del aislamiento o la ejecución de lavados periódicos del mismo, a veces sin ningún criterio técnico, lo que demanda una mano de obra considerable. Para el caso de la corrosión o de la destrucción parcial o total del aislamiento por las sobretensiones, las empresas no tienen más remedio que la sustitución del aislamiento cuando ocurre la falla, de manera general, o lo que se conoce como aplicación del mantenimiento correctivo. En los últimos años este problema ha tomado más importancia como consecuencia de la libre competencia de las empresas del sector eléctrico, ya que se necesita una optimización de las mismas y por ende una reducción de los costos de mantenimiento y mano de obra. El presente trabajo trata de exponer la relación estrecha que existe entre aislamiento y confiabilidad a partir de los nuevos paradigmas que se presentan en la actividad de mantenimiento, los cuales ofrecen algunos cambios interesantes en el entorno hasta ahora visto en esta dirección.

Palavras Chaves: Aisladores, mantenimiento, confiabilidad.

\section{INTRODUCCIÓN}

Un aislador eléctrico es un dispositivo cuya función es dar soporte rígido o flexible a los conductores o equipo eléctrico en el cual se encuentre instalado, aislando a éstos de tierra o de otros equipos. Generalmente, los conductores empleados en las líneas eléctricas aéreas son desnudos, por lo que deben ir aislados de las crucetas y apoyos correspondientes; por ello, se necesita el uso de 
los aisladores, que aíslen totalmente los conductores bajo tensión, de los apoyos que soportan la línea. La unión de los conductores a los aisladores y de los aisladores a los apoyos, se efectúa por medio de piezas metálicas denominadas herrajes.

En muchas ocasiones, a la hora de valorar dentro del proyecto de una instalación eléctrica el aislamiento, no se le da el valor correspondiente a la función que ellos ejecutan y la importancia que tiene dentro de la instalación. Si bien, por regla general, los aisladores no representan más allá del 4-5\% del costo total de la instalación, no por ello puede dejar de tenerse en cuenta que son una componente esencial en la integridad de las líneas y de la disponibilidad de energía.

La función esencial del aislador es evitar el paso de la corriente del conductor hacia el apoyo, pero en ocasiones, esta función no se cumple por cualquiera de las siguientes causas:

a) Perforación del aislador: Consiste en una falla de las propiedades dieléctricas en algún punto del interior del aislador que puede provocar su perforación.

b) Conductividad interior: Consiste en la conducción de una corriente de fuga a través del interior del aislador. Con los materiales que se emplean en la actualidad en la fabricación de aisladores, esta corriente resulta despreciable, excepto cuando está perforado parcialmente.

c) Descarga disruptiva a través del aire: Cuando la rigidez dieléctrica del aire que rodea al aislador disminuye hasta un valor crítico, se forma un arco eléctrico a través de la superficie del aislador, entre el soporte del mismo y el conductor que sostiene. Con la humedad, la rigidez dieléctrica del aire disminuye, y en el caso de la lluvia, las gotas de agua que se desprenden de la superficie del aislador toman el potencial del conductor, disminuyendo la distancia entre el soporte del aislador y el conductor.

d) Conductividad superficial: Conducción por el contorno de la parte exterior del aislador por aumento de su conductividad debido a la formación de una capa contaminante de polvo, arena, sales, entre otros, depositadas sobre la superficie del aislador cuando ésta se humedece.

No son pocas las veces en que la selección del aislamiento es tratada por áreas importadoras o de compra, cuando de hecho ellos son la clave para el transporte y distribución de energía desde las plantas de generación a los clientes finales. Existen varios criterios que pueden enunciarse y que de forma resumida representan algunas formas de atender el problema del aislamiento eléctrico en los proyectos de las instalaciones, por parte de algunas empresas:

1. En muchas ocasiones, para satisfacer la presión de los precios de adquisición, frecuentemente son aceptadas concesiones en el proyecto y selección de los aisladores.

2. La adquisición de nuevos modelos y tipos de aisladores es frecuentemente influenciada por "modas" en lugar de ser una decisión de carácter técnico, fundamentada en un análisis racional de desempeño a partir de experiencias internacionales y propias.

3. Los requisitos de mantenimiento de cada tipo y modelo de aislador deben incluir procedimientos de inspección comprobados y confiables, no solamente para detectar aisladores defectuosos o deteriorados, sino también para poder ofrecer condiciones de trabajo seguras cuando se trabaja de forma energizada, requisitos estos que no siempre son comprobados a la hora de utilizar nuevos proyectos de aisladores.
4. Por último, el costo de la vida útil del aislador debe ser un indicador de satisfacción para la adquisición, más que el propio precio inicial de adquisición.

Muchas veces el bajo precio de adquisición no representa ningún índice real de desempeño si los costos adicionales de operación y de mantenimiento no son considerados en la ecuación [1].

\section{LA SEGURIDAD Y LA CONFIABILIDAD: SU INTERRELACIÓN}

La seguridad y la confiabilidad son dos términos que muchas veces los ingenieros no los ven como elementos componentes de un sistema único. La calidad de la energía depende de una variedad de parámetros, uno de los cuales se relaciona con la integridad del aislamiento; la integridad del aislador, y sus condiciones de operación y mantenimiento, hacen que conceptos tales como seguridad y confiabilidad comiencen a entrelazarse.

Por lo general, la seguridad, en la mayor parte de las veces, se enfoca hacia la prevención de accidentes asociados a formas erróneas de proceder en una situación dada, Sin embargo, son pocas las veces que la seguridad se ve desde el punto de vista de la pérdida de la función de un elemento, que en composición de un sistema cualquiera, ejecuta una labor dada, y que en un momento dado puede poner en riesgo la vida del especialista de mantenimiento.

Por otro lado, la confiabilidad se enfoca normalmente, hacia cómo hacer que un sistema trabaje de forma correcta. Por regla general, cuando un sistema requiere un determinado nivel de seguridad y de confiabilidad, existirán siempre dos atributos actuando. Supongamos que se parte de una condición primaria que se tiene con relación a la seguridad y la confiabilidad, representada por el punto 1 de la Figura 1.

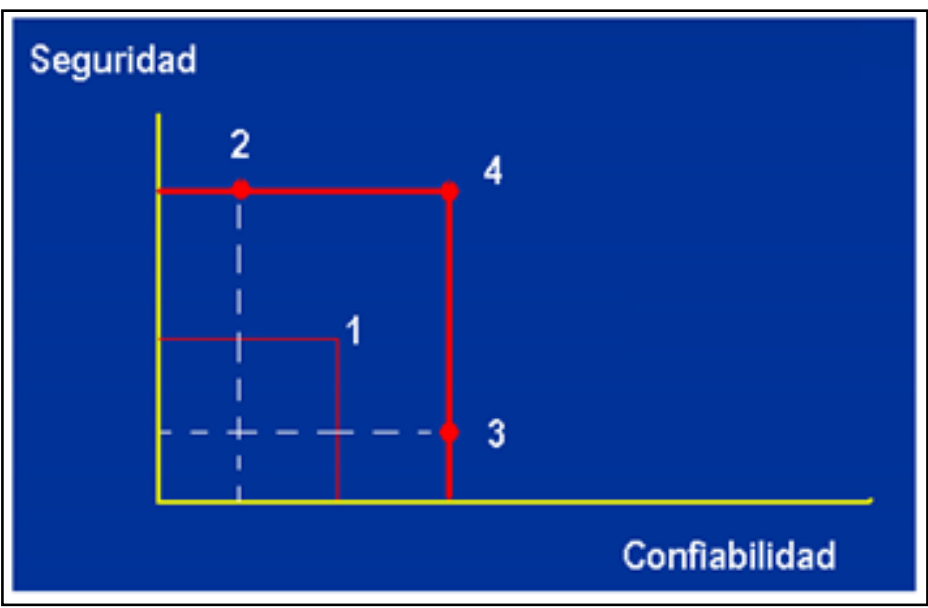

Figura 1: Relación entre seguridad y confiabilidad.

Fuente: Autor, (2018).

- En ocasiones se desea incrementar la seguridad de un sistema, pero ello implica disminuir la confiabilidad; el estado nuevo del sistema se localizará en el punto 2 . Un ejemplo de ello es cuando se hacen inversiones en medios de seguridad, pero no se ofrece un incremento en la capacitación de los operarios y técnicos para un correcto uso de esos nuevos equipos.

- En otras ocasiones se quiere aumentar la confiabilidad del sistema, pero sin analizar que ello puede provocar una disminución de la seguridad del mismo, lo que nos lleva al estado 
del punto 3. Un ejemplo de ello es cuando se realiza la sustitución del aislamiento eléctrico externo para disminuir las interrupciones, y no se hace una adecuada selección del mismo teniendo en cuenta las características del mantenimiento que se ejecuta.

- Lo ideal es llegar al punto 4, o sea, trabajar porque seguridad y confiabilidad vayan de la mano y se logre incrementar una con el mejoramiento de la otra. Un ejemplo de ello es cuando la empresa sustituye el aislamiento de las líneas eléctricas, a cualquier nivel, y junto con esta decisión se instruye al personal sobre las nuevas estrategias y procedimientos de mantenimiento.

La confiabilidad de una instalación, o de sus elementos componentes, es la responsable de la disponibilidad de la instalación. Es por esto que la disponibilidad de una línea de transmisión y/o distribución de energía eléctrica estará en dependencia, entre otros elementos, de la confiabilidad de los aisladores que la soportan.

La confiabilidad de la transmisión y/o distribución de energía eléctrica puede ser analizada a través de sistemas diferentes. Una de las formas mas aceptadas de medir la disponibilidad de un sistema tiene su base en las reglas de los 9 "nueves". Para un país en desarrollo, este índice puede ser tan bajo como del 90\%, (un "nueve"), mientras que determinadas empresas pueden exigir un 99,9999999\% de disponibilidad (9 "nueves"). La diferencia entre estas dos exigencias varía desde 36,5 días de interrupción por año hasta 1,9 ciclos (a una frecuencia de $60 \mathrm{~Hz}$ ) por año [1].

Algunos autores plantean, a la luz de estos requerimientos, que el desempeño de los aisladores debe estar dirigido a los siguientes aspectos:

- La calidad de los aisladores debe ser definida en el nivel de confianza adecuado, NO PUDIÉNDOSE PERMITIR el concepto de falla del aislamiento, no en la forma que hoy día se maneja.

- En caso de que ocurra cualquier incidente en la línea, la falla debe ser corregida inmediatamente, lo que significa que no se debe permitir la degradación permanente del material, como es la carbonización o tracking, que obligue a la sustitución de aisladores para la restauración de la energía.

- El tiempo para la detección de la falla se convierte en un elemento crítico, por lo que la localización inmediata de la falla se convierte en un requisito perentorio y hasta obligatorio; el tiempo que se invierte en encontrar donde hay un aislador fallado se convierte en uno de los principales costos.

- El trabajo de mantenimiento, en cualquiera de sus variantes, debe convertirse en una posibilidad ante la introducción de nuevos modelos y tecnologías de aisladores, lo que disminuye el tiempo de interrupción a los clientes y la necesidad de circuitos en lazos y de redundancia en los mismos, todo lo cual tiene su propio costo.

Desde el punto de vista del Mantenimiento no debe olvidarse que existen principios básicos que involucran los recursos humanos (elemento esencial), las tecnologías y el aspecto económico, tal y como se observa en la Tabla 1.
Tabla 1: Elementos vinculados a la actividad de mantenimiento.

\begin{tabular}{|c|c|c|}
\hline Temas Técnicos & Recursos Humanos & Campo Económico \\
\hline $\begin{array}{l}\text { - Servicios } \\
\text { - Productos } \\
\text { - Calidad de los } \\
\text { productos } \\
\text { - Métodos de trabajo de } \\
\text { mantenimiento } \\
\text { - Manejo óptimo de } \\
\text { materiales } \\
\text { - Control de todas las } \\
\text { actividades de } \\
\text { mantenimiento }\end{array}$ & $\begin{array}{l}\text { - Función de relaciones } \\
\text { externas } \\
\text { - Función de la } \\
\text { organización del } \\
\text { mantenimiento }\end{array}$ & $\begin{array}{l}\text { - Estructura de } \\
\text { mantenimiento } \\
\text { - Economía en la } \\
\text { gerencia del } \\
\text { mantenimiento } \\
\text { - Economía frente a la } \\
\text { producción }\end{array}$ \\
\hline
\end{tabular}

Fuente: [2].

Por otro lado, los paradigmas actuales del Mantenimiento han cambiado, lo que hace que también cambien las formas de ejecutarlo, y en ello ha incidido grandemente la introducción de nuevas tecnologías en la operación o producción de las empresas, tal y como se muestra en la Tabla 2.

$\mathrm{Si}$ se hace algunas reflexiones sobre como estos paradigmas cambian los conceptos sobre el aislamiento eléctrico a modo de ejemplo pueden verse 3 casos:

Sobre el paradigma 1- Es uno de los cambios que pudieran catalogarse como fundamental, pues evidentemente redirige el objetivo principal del mantenimiento, de la preservación del equipo o elemento, hacia la preservación de sus funciones.

¿Qué significa esto, pudiera cualquier persona preguntarse? Esto no significa otra cosa que si con anterioridad para una empresa eléctrica, hablando de aislamiento eléctrico, el mantenimiento estaba dirigido hacia mantener físicamente al aislador, hoy el mantenimiento está dirigido a diagnosticar si el mismo ha perdido sus propiedades lo cual lo limita a cumplir su función principal: aislar.

Sobre el paradigma 4- El Mantenimiento actual va dirigido hacia el análisis de los fallos y detectar sus causas, no analizar el equipo como tal.

Un equipo o elemento puede tener muchos años de trabajo y seguir cumpliendo su función, porque las condiciones a la cual ha estado operando son para las cuales se diseñó, y por tanto su vida útil es mayor; en contraposición, un equipo o elemento puede que al cabo de un tiempo relativamente corto deje de cumplir su función pues se puso a trabajar en un ambiente para el cual no estaba diseñado. En el caso del aislamiento, este concepto de Mantenimiento explica por si mismo que no es posible sustituir el aislamiento por modismos o porque se haya puesto viejo; es necesario analizar si sus características técnicas y constructivas son adecuadas para las condiciones de operación: si lo son, trabajará sin problemas, y en caso contrario, tendrá muchas dificultades y aparentemente, habrá envejecido.

Sobre el paradigma 9.- En el Mantenimiento actual nada está fuera del control y de las posibilidades de evitar que ocurra. El problema radica en detectar, con tiempo suficiente los fallos incipientes que puedan crear una situación de caos, y solucionarla con un buen Mantenimiento.

El aislamiento eléctrico no falla masivamente si no es por una mala selección del mismo; la experiencia dice que los fallos masivos de aislamiento han estado precedidos de:

- Falta de chequeo sistemático y de mantenimiento. 
- Decisiones arbitrarias que no tienen en cuenta las características técnicas y posibilidades de trabajar, del aislamiento, bajo las condiciones de operación que se le imponen.

Así mismo aparecen nuevos paradigmas que de una u otra forma van dirigidos a esclarecer la importancia del papel del hombre que trabaja directamente sobre el equipo y/o elemento en la ejecución exitosa del Mantenimiento y la responsabilidad de la gerencia de ofrecerle las herramientas necesarias para el mismo, así como a esclarecer que el trabajo de Mantenimiento no es un problema del área de Mantenimiento, sino de toda la empresa.

Tabla 2: Nuevos paradigmas para la actividad de Mantenimiento.

\begin{tabular}{|c|c|}
\hline No. & Paradigma moderno \\
\hline 1 & 1 mantenimiento se oc upa de la preservación de las funciones de los activos. \\
\hline 2 & El mantenimiento de rutina es para evitar, reducir o eliminar las consecuencias de los fallos. \\
\hline 3 & $\begin{array}{l}\text { El mante nimiento afecta todos los aspectos de efectividad del negocio: riesgo, seguridad, integridad del } \\
\text { medio ambiente, uso eficiente de la energí, calidad de producto y servicio al cliente. No solamente } \\
\text { disponibilidad de planta y costo. }\end{array}$ \\
\hline 4 & La mayoría de los fallos no son más probables cuando el equipo envejece. \\
\hline 5 & $\begin{array}{l}\text { Las decisiones sobre el control de fallos de los equipos casi siempre tendrán que ser tomadas con datos } \\
\text { inadecuados sobre razones de fallos. }\end{array}$ \\
\hline 6 & Hay cuatro tipos básicos de mantenimiento: Predic tivo - Preventivo - Correc tivo- Detec tivo. \\
\hline 7 & $\begin{array}{l}\text { La frecuencia con que se realizan las tareas "a-condición-de" debe basarse en la duración del período de } \\
\text { desarrollo de la falla (también conocido como "tiempo de demora hasta la falla" ó "intervalo P-F"). }\end{array}$ \\
\hline 8 & $\begin{array}{l}\text { En caso de ser técnicamente factible tanto una tarea de restauración o reemplazo a intervalos fijos } \\
\text { (preventiva), como una tarea “a condición de" (predictiva), ésta última es generalmente más económica y } \\
\text { efectiva a lo largo de la vida del activo. }\end{array}$ \\
\hline 9 & $\begin{array}{l}\text { La probabilidad de un fallo múltiple es, en buena medida, una variable manejable, especialmente en } \\
\text { sistemas protegidos. }\end{array}$ \\
\hline 10 & $\begin{array}{l}\text { Casi siempre es más costo-eficaz tratar de mejorar el comportamiento de un activo no confiable mejorando } \\
\text { la forma en que es operado y mantenido, revisando el diseño sólo si tal solución no logra el } \\
\text { comportamiento deseado. }\end{array}$ \\
\hline 11 & $\begin{array}{l}\text { Las políticas genéricas solamente deben a plicarse en activos idénticos cuyo contexto operacional, } \\
\text { funciones y parámetros de prestación deseados también sean idénticos. }\end{array}$ \\
\hline 12 & $\begin{array}{l}\text { Las polític as de mante nimiento deben ser establecidas por las personas que están más cerca de los activos. } \\
\text { La responsabilidad de la Dirección } \mathrm{y} \text { Gerencia es de proveer las he ramientas que les permitan tomar las } \\
\text { decisiones correctas y asegurar que esas decisiones se an sensatas y defendibles. }\end{array}$ \\
\hline 13 & $\begin{array}{l}\text { Un Programa de Mantenimiento sólo puede ser exitoso y perdurable si es desarrollado por } \\
\text { "Mantenimiento" y "Usuarios de los activos", trabajando juntos. }\end{array}$ \\
\hline 14 & $\begin{array}{l}\text { Los fabric antes y proveedores de equipos solamente pueden desempeñar un papel limitado -aunque } \\
\text { importante - en el desarrollo de programas de mantenimiento. }\end{array}$ \\
\hline 15 & $\begin{array}{l}\text { Los problemas del mantenimiento obtienen su mejor solución trabajando en dos etapas: cambiando la } \\
\text { forma en que las personas piensan y logrando que apliquen esa nueva manera de pensar, a la resolución de } \\
\text { problemas téc nicos de proceso - un paso porvez. }\end{array}$ \\
\hline
\end{tabular}

Fuente: [2].

Sobre el paradigma 13 - Si el personal de Mantenimiento no trabaja de forma coordinada con el área de operación los resultados de la explotación del equipo, y en consecuencia, el nivel de fallos, puede ir en incremento en lugar de disminuir, ya que acciones de operación pueden traer como consecuencia desgastes de los equipos, y acciones de mantenimiento pueden traer consecuencias para la operación de los mismos.

Por ejemplo, si una brigada de trabajo en caliente ejecuta un mantenimiento como el lavado de aislamiento con agua a presión, bajo el concepto de que el aislamiento está contaminado, si este es ejecutado en una zona donde la contaminación es del tipo químico o cemento, lo más probables es que las fallas se incrementen y por tanto se dificulte el trabajo del área de operación de la empresa. Aquí el problema radica en desconocimiento de la causa de los fallos y no en un mal mantenimiento.
Sobre el paradigma 14 - Anteriormente muchos mantenimientos se ejecutaban a partir de lo que decía el fabricante; la experiencia ha demostrado que no siempre es así, pues los ambientes y condiciones de trabajo cambian.

Por ejemplo, los aisladores poliméricos han demostrado, en unas regiones que son capaces de trabajar por períodos superiores a los 20 años, coincidiendo esto con lo que los fabricantes dicen. Sin embargo, en otras regiones, los mismos aisladores son incapaces de trabajar establemente en cuestión de meses, o como máximo se mantiene estables durante 5 años. Vale preguntarse entonces: ¿es que el fabricante estaba equivocado? Por regla general el problema está en que la empresa que los introdujo no evaluó, o no le dio al fabricante, todos los requerimientos de trabajo, y el tiempo, el implacable, le pasó factura al aislamiento y a la decisión tomada. Los aisladores, por regla general, no son malos, sino mal seleccionados. Los fabricantes de aisladores que se 
respetan no sacan al mercado un producto defectuoso, por regla general, pues pierden clientela.

En el caso del aislamiento convencional, ya sea esta de porcelana electrotécnica o de vidrio, estas propiedades y características han sido bien estudiadas. Sin embargo, la presencia de los aisladores sintéticos, o poliméricos por ejemplificar un caso, han cambiado algunas cuestiones que ya se daban por sentadas con el uso de los aisladores convencionales. Por tal razón la seguridad y la mantenibilidad de este tipo de aislamiento hay que enfocarlo de manera diferente y en ello incide mucho el nivel de conocimientos que sobre los mismos se tengan y las diferentes técnicas de mantenimiento y diagnóstico que se utilizan actualmente.

Aún cuando la inspección visual sigue siendo un método efectivo para muchos problemas que existen en el aislamiento, incluyendo la mala colocación o instalación del mismo en las estructuras y líneas, se ha dificultado mucho su aplicación con la presencia de los aisladores sintéticos, ya que muchos problemas no pueden ser detectados a larga distancia o con la ayuda de prismáticos desde debajo de las estructuras; hay que escalar la estructura o acercarse al aislamiento de alguna forma para poder observar los daños.

Partiendo de esta consideración puede decirse que hasta la fecha no existe todavía una técnica normalizada para inspeccionar el aislamiento sintético en operación; diversas organizaciones en conjunto con las compañías eléctricas han investigado varias técnicas, aplicables a aisladores energizados. En estos momentos lo más cercano a permitir una evaluación del aislamiento sintético ubicado en las líneas aéreas de transmisión y distribución es una guía visual para la inspección desarrollada por el STRI, conocida como Guía 5 (ver Figura 2).

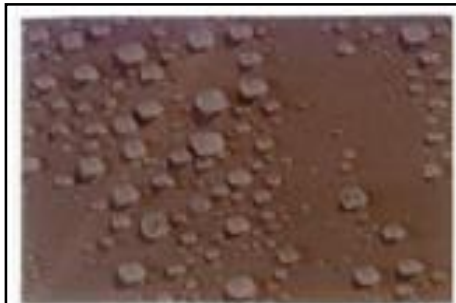

$\mathrm{HC} 1$

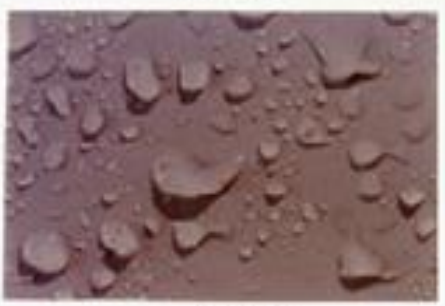

$\mathrm{HC} 3$

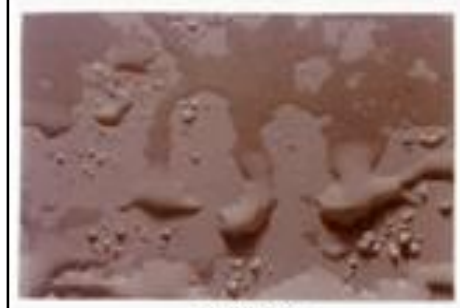

HC 5

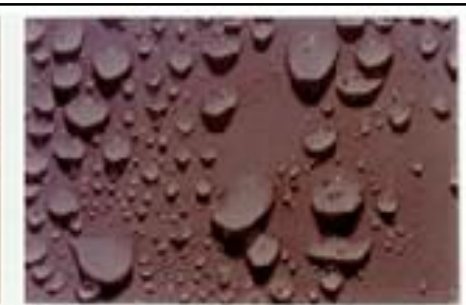

$\mathrm{HC} 2$

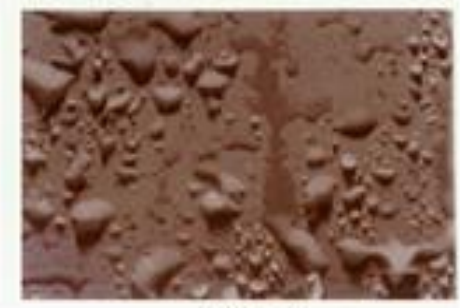

$\mathrm{HC} 4$

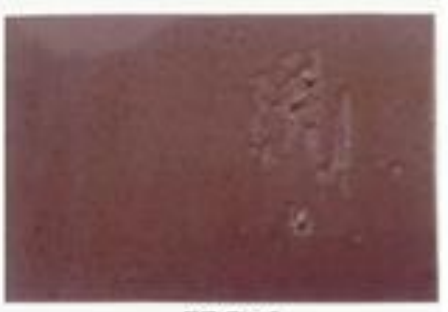

HC 6
Figura 2. Método STRI para determinar deterioro de la propiedad de hidrofobicidad en el aislamiento sintético.

Fuente: Autor, (2018).
El método STRI permite evaluar, de una manera muy sencilla, la pérdida de hidrofobicidad del aislamiento sintético, que es una de sus propiedades fundamentales, a partir de una valoración visual de su superficie. La pérdida de hidrofobicidad es una medida de que el aislador está perdiendo sus características constructivas y por tanto puede fallar en cualquier momento, ante condiciones de alta contaminación.

Su desventaja radica en que en aislamientos del tipo epóxido, esta evaluación no representa lo mismo que en los aislamientos no epóxidos, y por tanto no es una medida generalizable para todos los aisladores sintéticos.

Nuevas técnicas, que permitan realizar un trabajo más seguro en las actividades de mantenimiento que normalmente se realizan, ante la presencia de diferentes modelos y tipos de aisladores se han ido desarrollando, más aún no se posee un nivel de seguridad que permita avalarlas desde el punto de vista de su generalización. Ente estas nuevas técnicas o tecnologías pueden mencionarse cuatro que al parecer pueden ofrecer un camino muy útil para el diagnóstico del estado del aislamiento bajo estas nuevas condiciones: la cámara de corona, el equipo para medir la emisión acústica, la cámara de descargas parciales y la termografía infrarroja (ver Figura 3).

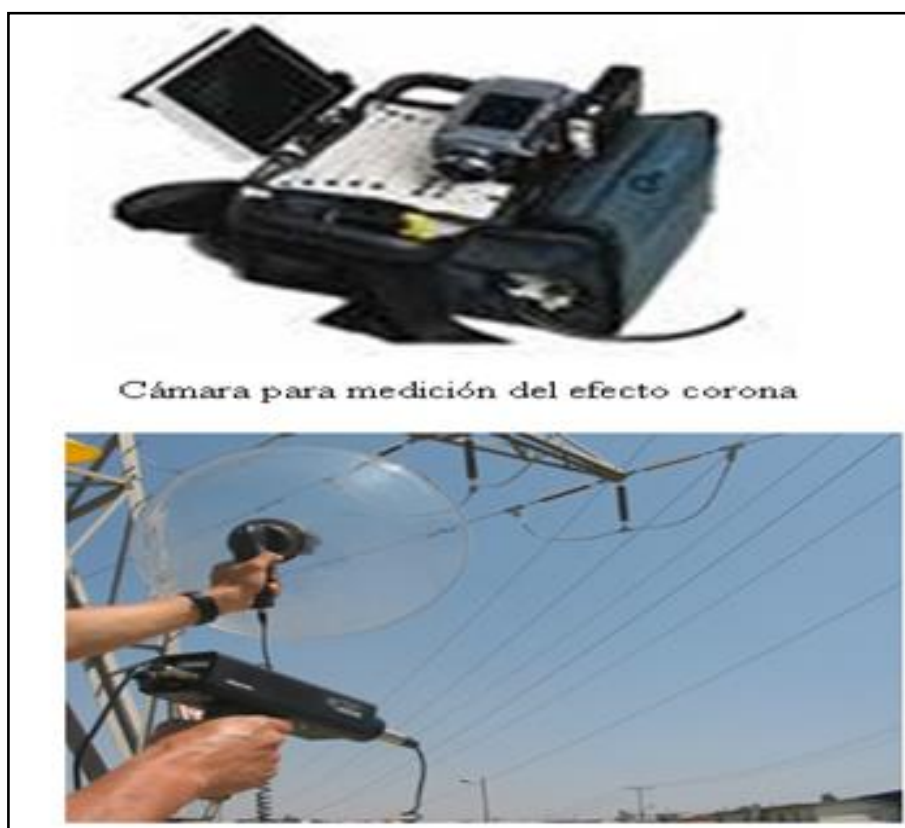

Equipo para medición de emisiones acústicas

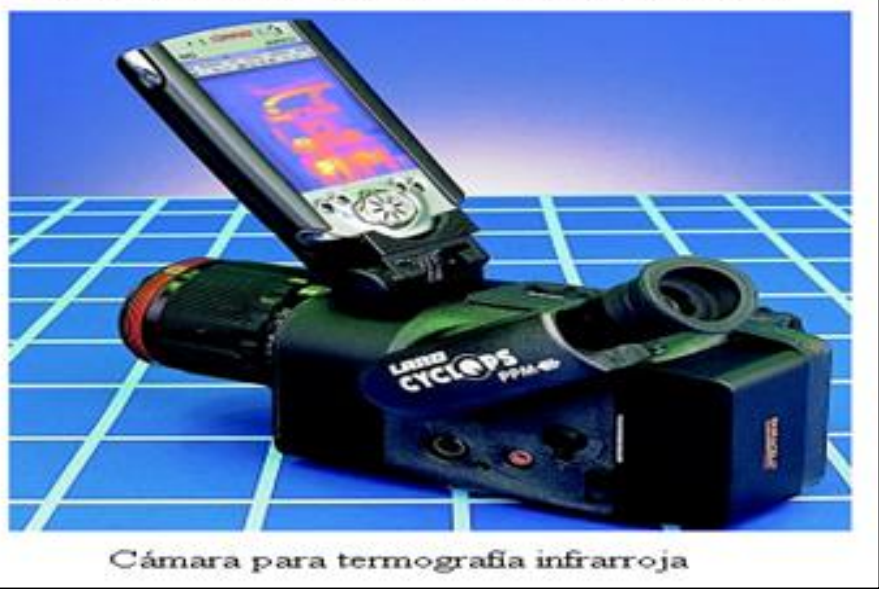

Figura 3. Nuevas tecnologías para el mantenimiento. Fuente: Autor, (2018). 
El detector de corona es muy efectivo pero costoso, mientras que la detección acústica cubre un amplio campo de posibilidades y a su favor está que es mucho más barata y asequible a muchas empresas; su desventaja radica en que para un especialista no experto las señales de ruido pueden ser confundidas con las que producen las descargas de corona.

La detección de las descargas parciales permite definir internamente si existen problemas en el material aislante y con una buena formación del especialista puede llegar a ofrecer puntos de análisis muy importantes para las fallas internas del aislamiento, mientras que la termografía infrarroja es una herramienta muy importante bien empleada, pero realmente tiene el problema de que la generalización e interpretación de fallas incipientes es muy difícil, pues lo que en un lugar puede ser síntoma de un problema, en otro representa otra situación.

De forma general, puede decirse que no se ha dejado de trabajar por la mejora de las características y comportamiento de los aisladores ante la contaminación, incluyendo tanto la búsqueda de nuevas vías para mejorar el comportamiento del aislamiento tradicional (de vidrio y porcelana) como la mejora de los materiales sintéticos, así como en el desarrollo de nuevas técnicas y métodos de detección de su deterioro, como una vía de incrementar la seguridad y confiabilidad de su uso.

Una de las vías que se ha estado manejando para minimizar los efectos de la contaminación sobre el aislamiento externo, y que desde hace algunos años tiene alcance comercial, es el uso de la silicona vulcanizada a temperatura ambiente (RTV SR, según sus siglas en inglés) para cubrir aisladores de vidrio y porcelana. Los recubrimientos empleados actualmente son el resultado de más de treinta años de desarrollo y utilización, sobretodo en ambientes de alto grado de contaminación, y como resultado de un estudio realizado [3] se ha podido corroborar que aun cuando la RTV SR empleada en la cubierta de los aisladores de porcelana y vidrio se puede ver afectada por diferentes factores que de forma general no afectarían a estos aisladores sin su presencia, la hidrofobicidad de este material resulta de gran importancia para combatir los efectos nocivos de la contaminación de origen natural o industrial sobre los sistemas eléctricos, siempre y cuando la formulación y aplicación de la RTV SR sea realizada de forma correcta.

En esta dirección, una nueva tecnología parece abrir un campo muy interesante: la nanotecnología. La nanotecnología es un campo, de las ciencias aplicadas, dedicado al control y manipulación de la materia a una escala menor que un micrómetro, es decir, a nivel de átomos y moléculas (nanomateriales). Lo más habitual es que tal manipulación se produzca en un rango de entre uno y cien nanómetros.

El campo de la nanotecnología ha emergido como uno de los más activos en las áreas tecnológicas a nivel mundial, y el interés de lo que se ha dado por llamar los nano dieléctricos (dieléctricos conformados a partir de nano elementos) ha crecido rápidamente dado su potencial para una nueva generación de materiales aislantes para alta tensión (AT) con propiedades únicas [4].

Es bien conocido que se pueden mejorar las propiedades de los polímeros adicionando partículas sólidas a las resinas termoplásticas, pero también se conoce que muchas o varias propiedades de los compuestos originales pueden ser cambiadas, $\mathrm{y}$ es algo que debe esclarecerse totalmente al trabajar y caracterizar a los nanos dieléctricos [5,6].

Por otro lado, se ha planteado la posibilidad de trabajar en dos direcciones principales, en la búsqueda de incrementar la confiabilidad de estos elementos: el uso de los nanos dieléctricos para ayudar a retardar el envejecimiento prematuro de los materiales dieléctricos sólidos debido a los efectos de la contaminación, los rayos ultravioletas, la humedad y el campo eléctrico, actuando de forma diferenciada o conjunta, y el uso de tecnología nano que permita monitorear el nivel de descargas parciales o la presencia de puntos calientes desde el interior del material aislante [7].

Como uno de los resultados asociados al proyecto mencionado en [7], se logró proponer y elaborar un recubrimiento sobre la base de nanomateriales que aseguró mejorar las características de comportamiento ante la contaminación de aisladores de vidrio y porcelana [8], a partir del estudio de influencia que tiene el agregar nanosílice como carga a la goma de silicona vulcanizada a temperatura ambiente (RTV SR, por sus siglas en inglés) que es empleada para cubrir aisladores de vidrio o de porcelana. Este trabajo permitió obtener la dispersión de la nanosílice en la RTV SR por medio de microscopía electrónica de barrido (MEV, por sus siglas en inglés), se midió la permitividad de la RTV SR con y sin nanosílice para varias frecuencias y se evaluó la influencia que tiene la nanosílice en la resistencia a la erosión. Además fueron medidas las corrientes de fuga durante ensayos de niebla salina a aisladores de vidrio pintados con estos recubrimientos, analizando también la pérdida de hidrofobicidad que ocurre durante el ensayo y su recuperación una vez finalizado el mismo.

Otros artículos relacionados con este proyecto y estudio pueden verse en [9-12].

\section{CONCLUSIONES}

En lo referente a los aisladores, existe una enorme acumulación de eventos a escala internacional que demuestran que cada vez que una falla ocurre su costo inducido es siempre más elevado que el costo original de la adquisición de los aisladores. Por tal razón la selección de un aislamiento adecuado es muy importante para disminuir los costos ya en explotación.

Es por eso que el uso de los aisladores poliméricos no puede ser visto sólo desde el punto de vista de sus nuevas características o ventajas, independientemente de los resultados de los ensayos de campo y laboratorio. Así mismo hay otros factores que deben ser tenidos en cuenta a la hora de su uso y selección ante los aisladores tipo convencional (porcelana y vidrio) y uno de ellos es el mantenimiento.

Un buen procedimiento de inspección, junto a la calidad del aislamiento, y la aplicación de nuevas técnicas y tecnologías asociado a procedimientos de mantenimiento de trabajo seguro, es una de las garantías de disminuir los costos por fallos en las líneas de transmisión de energía eléctrica y los riesgos en el trabajo con este nuevo tipo de aislamiento.

\section{REFERENCIAS}

[1] J.M. George, La íntima relación entre el costo de operación de las líneas de transmisión y la selección del aislador, SEDIVER, documento A1.0435CTS05. 
[2] L. A. Mora G, Mantenimiento estratégico para empresas industriales o de servicios ,2a .Edición, Marzo 2007.

[3] I. Pérez, E. Guedes, M. Castro, “Algunas consideraciones sobre la silicona vulcanizada a temperatura ambiente empleada en el aislamiento externo., X Congreso Latinoamericano y VII Iberoamericano en Alta tensión y Aislamiento Eléctrico, Hotel Meliá Confort Habana, La Habana, Cuba, Septiembre 2011.

[4] M.F. Fréchette, C. W. Reed, H. Sedding, "Progress, Understanding and Challenges in the Field of Nanodielectrics", IEEE An. Rep. Conf. Electr. Insul. Diel. Phen. (CEIDP), pp. 162-165, 2005.

[5] Y. Cao, P.C. Irwin, K. Younsi, " The Future of Nanodielectrics in the Electrical Power Industry', IEEE Transactions on Dielectrics and Electrical Insulation Vol. 11, No. 5; October 2004, pp. $797-807$.

[6] Y. Cao and P. C. Irwin, "Electrical Conduction in Polyimide Nanocomposites', IEEE Conf. Electr. Insul. Dielectr. Phenomena, pp. 116_119, Albuquerque, USA, 2003.

[7] M. Castro, J. A. Martínez, “' Nano dieléctricos: aplicación para la solución a problemas del aislamiento en el sector eléctrico", propuesta de proyecto de investigación, Marzo 2009.

[8] I.Pérez, D.Cibele, M.Castro, E.Guedes, A.Germano, "Nanosílice como carga en la RTV SR usada para cubrir aisladores", Revista Ingeniería Energética Vol. XXXIII No.1/2012, Enero-Abril, p.9-15. ISSN: 1815 - 5901

[9] E. Coser, C. A. Ferreira, J.M.G. Angelini, B. J. G. de Aragão, y I. P. Almirall, «Mechanical Analysis of Silicone Rubber used on the Cover of Polymeric Insulators», IEEE Latin America Transaction, vol. 8, nº 6, pp. 653-657, dic. 2010.

[10] E. Coser, A. Ferreira, C. Giacomini A., J. Marí, I. Pérez. "Study of kinetic behavior of silicone rubber used on the cover of polymeric insulators." Revista IEEE América Latina, Vol. 9, No. 7, December 2011, p. 1073 - 1078. ISSN 1548-0992.

[11] I. Pérez, E. Guedes, D. Cibele, M. Castro, A. C. F. M. Costa, y A. Germano, «Efecto de la variación de la ATH como carga en la cubierta polimérica de aisladores de vidrio», presented at the Simpósio Brasileiro de Sistemas Elétricos (SBSE 2012), Goiás, Brasil, 2012.

[12] I.Pérez, Empleo conjunto de de cargas micrométricas y nanométricas para mejorar el desempeño de la silicona vulcanizada a temperatura ambiente empleada para recubrir el aislamiento externo, tesis presentada en opción al grado científico de Dr. en Ciencias Técnicas, CIPEL, CUJAE, La Habana, Enero de 2014. 